\title{
Aceptabilidad por el conejo de la harina de coco desgrasada entre diferentes fuentes de alimento
}

\section{Rabbit Acceptability of Defatted Coconut Flour Among Different Food Sources}

\author{
Yanixi Acosta Acosta' \\ Ángel Luis La O Michel ${ }^{2}$ \\ Manuel Valdivié Navarro ${ }^{3}$ \\ Nelson Nixon Betancourt Santos ${ }^{4}$ \\ Yudelkis Villalón Moracen ${ }^{5}$
}

DOI: https://doi.org/10.19053/01228420.v18.n1.2021.12521

RESUMEN: Con el propósito de determinar el grado de aceptabilidad de la harina de coco desgrasada suministrada como alimento único e incorporado en diferentes niveles en la dieta para conejos, fueron realizados dos experimentos en los que se emplearon diseños bloques al azar. En el primer experimento los tratamientos fueron: $T_{1}$ harina de maíz, $T_{2}$ harina de alfalfa y $T_{3}$ harina de coco desgrasada; para el segundo, los tratamientos consistieron en el suministro de las dietas con la inclusión de 0,10,20,30 y $40 \%$ de harina de coco desgrasada. En los dos estudios se utilizaron conejos machos de la raza chinchilla de 80 días de edad, con un peso promedio de $2150 \pm 103 \mathrm{~g}, 21$ y 35 animales respectivamente. Se observó una conducta similar en las variables intento de consumo y consumo de alimentos, tanto cuando se suministró la harina de coco desgrasada como alimento único como cuando se mezcló con otros ingredientes. Los animales realizaron intentos de consumo durante casi las $24 \mathrm{~h}$, con los mayores intentos de consumo en el horario nocturno. Los animales muestran la mayor aceptación por las dietas del 10\% de inclusión de harina de coco desgrasada. Los resultados sugieren que la harina de coco desgrasada es un subproducto agroindustrial con potencialidades para ser incorporada en las dietas de conejos y sustituir alimentos convencionales tradicionalmente empleados en los piensos elaborados para esta especie.

PALABRAS CLAVE: subproductos agroindustriales, alimentación, conejos, prueba de palatabilidad.
ABSTRACT: To determine the acceptability of defatted coconut flour supplied as the sole feed and incorporated at different levels in the rabbit diet, two experiments were performed in which random block designs were used. In the first experiment the treatments were: $T_{1}$ corn flour, $T_{2}$ alfalfa flour, and $T_{3}$ defatted coconut flour. In the second, the treatments consisted of supplying the diets with the inclusion of defatted coconut flour in growing percentage $(0,10,20,30$ and $40 \%)$. In both studies Chinchilla breed male rabbits of 80 days old were used, weighting an average of 2,150 $\pm 103 \mathrm{~g}, 21$ and 35 rabbits respectively. Variables attempt to consume and food consume showed similar results, both in defatted coconut flour supplied as the sole feed and when mixed with other ingredients. Animals made consumption attempts for nearly 24 hours and the highest consumption attempts were observed at night. Animals showed the highest acceptance for diets of $10 \%$ defatted coconut flour inclusion. The results suggest defatted coconut flour is an agro-industrial by-product with potentiality to be included in rabbit diets and to substitute traditional feed used for this specie.

KEYWORDS: agro-industrial by product, feed, rabbits, palatability test.

FECHA DE RECEPCIÓN: 09 de julio de 2020 FECHA DE APROBACIÓN: 17 de julio de 2020

COMO CITAR ESTE ARTíCULO: Acosta Acosta, Y., La O Michel, Ángel, Valdivié Navarro, M., Betancourt Santos, N., \& Villalón Moracen, Y. (2021). Aceptabilidad por el conejo de la harina de coco desgrasada entre diferentes fuentes de alimento. Cien. Agri. 18(1): 76-85. https://doi.org/10.19053/01228420. v18.n1.2021.12521

1 Universidad de Guantánamo. Facultad Agroforestal. Avenida Che Guevara km 1.5 Carretera Jamaica, Guantánamo, Cuba, CP: 95 100. yanixi@cug.co.cu (D) https://orcid.org/0000-0001-8765-0046X

2 Universidad de Guantánamo. Facultad Agroforestal. Avenida Che Guevara km 1.5 Carretera Jamaica, Guantánamo, Cuba, CP: 95 100. nolo@cug.co.cu (D) https://orcid.org/ 0000-0002-7620-411x

3 Universidad Agraria de La Habana "Fructuoso Rodríguez". Instituto de Ciencia Animal Mayabeque. Cuba. mvaldivie@ica.co.cu (iD) https://orcid.org/0000-0002-8858-0307

4 M.Sc. Oficina Nacional de Inspección Estatal. Carretera de Jamaica Km 6 1⁄2. Guantánamo, Cuba, CP: 95 100. nelson.betancourt@hab.alinet.cu (D) https://orcid.org/0000-0002-8939-8005

5 M.Sc. Universidad de Guantánamo. Facultad Agroforestal. Avenida Che Guevara km 1.5 Carretera Jamaica Guantánamo, Cuba, CP: 95100. yudelkis@cug.co.cu@ (D)tps://orcid.org/0000-0002-2344-9527 


\section{Introducción}

La exploración de alternativas de alimentación animal en la producción de conejos acordes con las condiciones de países tropicales es tema de interés. Los esquemas de alimentación tradicionales se han basado en el uso de ingredientes dietéticos de origen vegetal, fundamentalmente soya, cereales y alfalfa, cultivos que pueden ser superados desde el punto de vista agronómico por otros mejor adaptados al medio y que no son requeridos para la alimentación humana. Esta situación estimula la búsqueda de nuevas materias primas alimentarias, con la finalidad de generar patrones de producción ajustados a la realidad social y económica del entorno en que se encuentran (Nieves \& Terán, 2006).

De esta forma, se establecerían estrategias alimentarias para conejos sobre la base de los recursos disponibles en los trópicos, con una adecuada utilización que promueva la sustentabilidad de los sistemas de producción (Nieves et al., 2011). Para ello, los estudios deben encaminarse al conocimiento de las cualidades específicas de los alimentos para esta especie, incluyendo palatabilidad, digestibilidad, contenido nutricional y los efectos sobre el funcionamiento intestinal y las enfermedades entéricas (Valverde, 2010).

En condiciones locales se ha desarrollado una serie de pruebas de aceptabilidad con la intención de explorar posibilidades de incorporación de estas fuentes alimenticias para conejos. Nieves et al. (2004b) estudiaron la inclusión de 30 y $40 \%$ de Leucaena leucocephala y Arachis pinto, mediante sustitución en una dieta basal para conejos de engorde con diferencias estadísticas $(p<0,05)$ y observaron mayor consumo y aceptación en las dietas que contenían L. leucocephala, aunque la inclusión de A. pintoi no implicó rechazo de las dietas.

Existe gran cantidad de residuos que se generan de la actividad agrícola que actualmente son materia de estudio con el fin de emplearlos como fuentes alternativas que produzcan rentabilidad en la alimentación de animales (Duban et al., 2012) En la región de Baracoa (Cuba), los subproductos más abundantes en función de los volúmenes que se producen son la pulpa de café, la cáscara de cacao y la harina de coco.

La harina de coco desgrasada, residuo obtenido de la extracción del aceite de coco por medio de prensado (expeller), es un alimento rico en proteína (19-20\%) según Tamilnadu et al. (2010) y Stein et al. (2015), grasas (3.0\%) y en fibra que, precisamente por esto, puede ser usado en raciones de conejos.

En este sentido, los estudios sobre la aceptabilidad de la harina de coco en la dieta de conejos aún son insuficientes; las posibilidades de su utilización en los sistemas de alimentación del conejo se basan en que es un animal herbívoro no rumiante cecofuncional y practicante de la cecotrofia, que utiliza 
alimentos fibrosos con relativa eficiencia y consigue ajustar el consumo en función de la concentración energética de la dieta.

El objetivo del presente estudio fue determinar el grado de aceptabilidad de la harina de coco desgrasada como alimento único e incorporado en diferentes niveles en la dieta de conejos raza chinchilla en Cuba.

\section{Materiales y Métodos}

La investigación se realizó en el Centro de Estudio de Tecnologías Agropecuaria y Forestal (CETAF) perteneciente a la Facultad Agroforestal adscrita a la Universidad de Guantánamo, el cual está ubicado en la región geográfica denominada Valle de Guantánamo, con coordenadas norte: 173300 m, este: 667750 m, a una altura de 83 m s.n.m. a 800 m al sur del poblado capital del municipio El Salvador.

La zona presenta un clima tropical de sabana, según la clasificación de Köppen. En general se caracteriza por temperaturas entre 27 y $29{ }^{\circ} \mathrm{C}$ promedio anual, precipitación anual relativamente pobre, con una distribución estacional muy marcada: presenta más del 60 \% del acumulado anual en el período mayo-octubre, conocido como período lluvioso. En toda su extensión presenta tres meses con precipitación media inferior a 60,0 mm.

La investigación se dividió en dos fases. Una primera fase donde se emplearon 21 conejos machos de la raza chinchilla de 80 días de edad, con peso promedio de $2150 \pm 103 \mathrm{~g}$, distribuidos en un diseño en bloques al azar tomando los días como bloques con tres repeticiones y siete réplicas; se aplicaron tres tratamientos: $T_{1}$ harina de maíz, $T_{2}$ harina de alfalfa $y T_{3}$ harina de coco desgrasada.

En la segunda fase se utilizaron 35 conejos machos de la raza chinchilla, de 80 días de edad, con peso promedio de $2150 \pm 101 \mathrm{~g}$, distribuidos en un diseño en bloques al azar, tomando los días como bloques con cinco réplicas y siete repeticiones; los tratamientos consistieron en el suministro de las dietas con la inclusión de 0, 10, 20, 30 y $40 \%$ de harina de coco desgrasada (Tabla 1). En ambos experimentos las variables controladas fueron los intentos de consumos y el consumo total de alimentos.

Los experimentos tuvieron una duración de nueve días, siete de adaptación y tres de muestreo; los animales fueron colocados individualmente en jaulas metálicas. Los alimentos fueron suministrados a razón de $100 \mathrm{~g}$ con acceso libre por los animales las 24 h (cafetería) según Nieves (2005), en tres y cinco comederos para la fase I y II respectivamente (Figura 1 ejemplo del experimento de la fase 2), siempre en el horario de la tarde, el agua fue suministrada ad libitum. 
TABLA 1. Ingredientes utilizados en las dietas evaluadas

\begin{tabular}{|c|c|c|c|c|c|}
\hline \multirow[b]{2}{*}{ Ingredientes } & \multicolumn{5}{|c|}{ Inclusión de harina de cocos nucifera L (\%) } \\
\hline & 0 & 10 & 20 & 30 & 40 \\
\hline Harina de maíz & 24,72 & 23,99 & 23,05 & 16,65 & 7,80 \\
\hline Harina de alfalfa & 55,33 & 50,04 & 45,84 & 46,33 & 47,96 \\
\hline Harina de soya & 12,87 & 10,11 & 6,85 & 2,85 & 0,00 \\
\hline Harina de cocos nucifera L. & 0,00 & 10,00 & 20,00 & 30,00 & 40,00 \\
\hline Fosfato dicálcico & 1,06 & 0,90 & 0,73 & 0,58 & 0,45 \\
\hline Carbonato de calcio & 0,00 & 0,00 & 0,00 & 0,00 & 0,17 \\
\hline Sal común & 0,30 & 0,30 & 0,30 & 0,30 & 0,30 \\
\hline DL-Metionina & 0,12 & 0,12 & 0,12 & 0,12 & 0,12 \\
\hline L-Lisina & 0,00 & 0,04 & 0,11 & 0,17 & 0,20 \\
\hline Premezcla & 2,00 & 2,00 & 2,00 & 2,00 & 2,00 \\
\hline Zeolita & 1,50 & 1,00 & 1,00 & 1,00 & 1,00 \\
\hline Aceite de coco & 2,10 & 1,50 & 0,00 & 0,00 & 0,00 \\
\hline
\end{tabular}

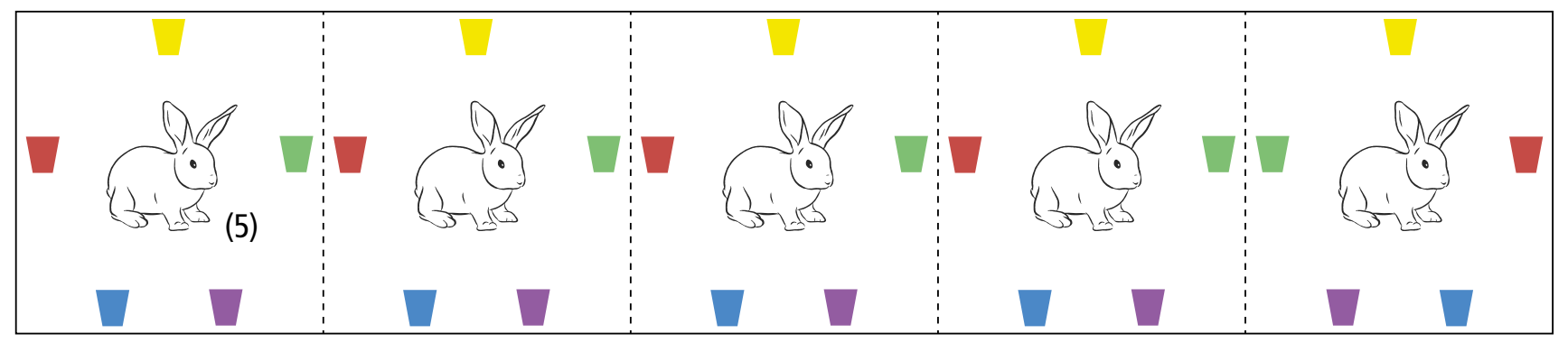

FIGURA 1. Esquema de la distribución de los animales y comederos en las jaulas.

Los intentos de consumo se obtuvieron por observación directa durante 72 h. El período de observación y registro de los intentos de consumo se realizó durante 10 minutos cada dos $\mathrm{h}$. Se observó el comedero y se registraron las veces que los animales consumieron el alimento. El consumo de alimento se determinó por diferencias entre el suministro y el rechazo.

El análisis estadístico de los intentos de consumos se realizó mediante la prueba no paramétrica de rangos múltiples de Kruskal-Wallis. En caso de obtener diferencias significativas, se hizo la comparación múltiple a través del estadístico $U$ de Mann-Whitney para un nivel de significación $(p<0.05)$. Para la evaluación del grado de aceptabilidad de los alimentos a través del consumo, se aplicó un análisis de varianza simple.

Para la evaluación del efecto del grado de aceptabilidad de la harina de coco desgrasada con niveles crecientes en las dietas a través de los intentos y el consumo de alimento, se aplicó un análisis de varianza simple. Para todos los casos, las diferencias entre las medias se determinaron con la aplicación de la prueba de Duncan (1955). 


\section{Resultados y Discusión}

El patrón de consumo de los conejos considerando el total de intentos de consumo de los alimentos ofertados (harina de maíz, alfalfa y harina de coco desgrasada), se muestra en la Figura 2. Se observa que los animales llevaron a cabo intentos de consumo durante casi las $24 \mathrm{~h}$, con pico de consumo al amanecer (5-7 h) con un descenso importante en los horarios de 11-15 h, a partir de las $17 \mathrm{~h}$ se inicia una nueva intensidad de intentos de consumo llegando a su máximo entre las 21 y $23 \mathrm{~h}$, lo cual significa que los animales mostraron una tendencia de consumo hacia el horario nocturno.

Esta conducta de consumo de alimento obtenida en el experimento está en correspondencia con las características de la especie en su vida natural y es propia del instinto de conservación de la especie ante los ataques de sus enemigos naturales. Resultados similares fueron reportados por Ferreira et al. (2008), quienes aseguran que los conejos incrementan el consumo de alimento en el horario nocturno, coincidiendo además con las horas más frescas, momento en el cual aprovechan más los concentrados y forrajes.

De esta forma, las condiciones ambientales, y en particular la temperatura, inciden marcadamente en el consumo de alimento. Al respecto, La O (2007), evaluando la aceptabilidad de cuatro forrajes, obtuvo que la conducta de los conejos en relación con el patrón de consumo fue similar a lo encontrado en el presente trabajo; la mayoría de los intentos de consumo por los animales se realizaron en los períodos de 17:00-21:00 $\mathrm{h}$ y de 5:00-9:00 $\mathrm{h}$.

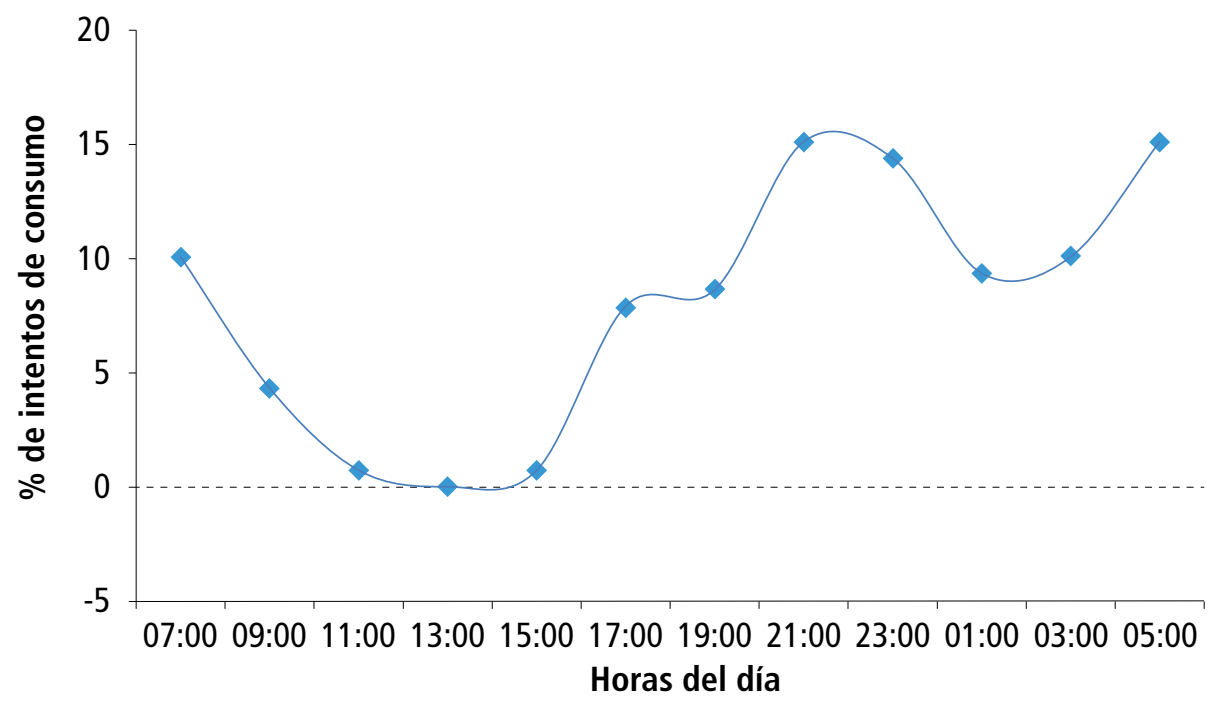

FIGURA 2. Patrón de intento de consumo de alimentos por los conejos durante $24 \mathrm{~h}$ donde se ofertaron harinas de maíz, alfalfa y coco.

Además, La O (2007) observó que en el período más caluroso del día solo se efectúa el 10,22 \% de los intentos, lo que corrobora el efecto negativo de 
las altas temperaturas en el consumo voluntario de los animales, valores que superan los intentos de consumo obtenidos en este trabajo, lo que pudo haber ocurrido por el tipo de alimento utilizado (harina), con estas condiciones los conejos evidencian necesidad de hiperventilación e hidratación y otros cambios conductuales que equivalen a la disminución del consumo de alimentos.

Los resultados relacionados con los intentos de consumos reflejados en la Tabla 2, refieren que los conejos tuvieron mayor preferencia por la harina de maíz, seguida por la harina de alfalfa y por último la harina de coco, según se muestra en los resultados de la prueba de rangos, donde se observó que los intentos de consumos fueron mayores para la harina de maíz en los horarios de 7, 19 y $21 \mathrm{~h}$, sin diferencias con la harina de alfalfa, mientras que en los horarios $9,15,17$ y 5 h no hubo diferencias entre la harina de maíz y alfalfa ni de esta última con la harina de coco.

La baja preferencia de la harina de coco desgrasada comparada con la harina de maíz y la alfalfa, podría estar muy relacionada con el aspecto a quemado que se observó en la harina de coco, ocasionado por el excesivo calor al cual es sometida, que, si bien favorece su conservación, puede modificar su aroma, olor y sabor, haciéndolo menos apetecible para el conejo.

Esta conducta de los conejos ante la harina de coco desgrasada sugiere que los animales necesitan de un período más prolongado para la adaptación a este alimento; al respecto Launchbaugh y Provenza (1991) señalan que los animales necesitan tiempo para cambiar los patrones de consumo alimenticio a los que están acostumbrados y esto lo realizan paulatinamente mediante la neofobia.

TABLA 2. Rangos promedios de intentos de consumo de los alimentos evaluados durante $24 \mathrm{~h}$

\begin{tabular}{|c|c|c|c|c|c|}
\hline \multirow[b]{2}{*}{ Horas } & \multicolumn{3}{|c|}{ Rangos promedios } & \multicolumn{2}{|c|}{ Significación } \\
\hline & H. de alfalfa & H. de maíz & H. de coco & Alimentos & Días (bloques) \\
\hline 7 & $30,1^{\mathrm{a}}$ & $25,3^{a}$ & $13,6^{b}$ & 0,001 & 0,098 \\
\hline 9 & $28,0^{a}$ & $24,2^{\mathrm{ab}}$ & $16,8^{b}$ & 0,033 & 0,025 \\
\hline 11 & 24,2 & 24,8 & 19,9 & 0,277 & 0,131 \\
\hline 13 & 21,5 & 26,1 & 21,5 & 0,171 & 0,291 \\
\hline 15 & $27,6^{a}$ & $23,6^{\mathrm{ab}}$ & $17,8^{b}$ & 0,037 & 0,059 \\
\hline 17 & $29,0^{\mathrm{a}}$ & $24,8^{\mathrm{ab}}$ & $15,2^{b}$ & 0,009 & 0,023 \\
\hline 19 & $28,8^{a}$ & $27,1^{\mathrm{a}}$ & $13,0^{b}$ & 0,001 & 0,018 \\
\hline 21 & $27,0^{\mathrm{a}}$ & $28,1^{\mathrm{a}}$ & $13,9^{b}$ & 0,003 & 0,062 \\
\hline 23 & 26,6 & 20,6 & 21,8 & 0,348 & 0,073 \\
\hline 1 & 24,6 & 24,4 & 20,0 & 0,420 & 0,805 \\
\hline 3 & 24,6 & 26,1 & 18,4 & 0,119 & 0,311 \\
\hline 5 & $31,1^{\mathrm{a}}$ & $23,0^{\mathrm{ab}}$ & $14,8^{b}$ & 0,002 & 0,042 \\
\hline
\end{tabular}

$a, b, c$ Letras diferentes en la misma fila indican diferencias significativas por $U$ de Mann-Whitney $(p<0,05)$ 
De Blas y Mateos (2010) mencionan que dietas nuevas suministradas a los conejos en libre elección no son aceptadas inmediatamente, pero su consumo puede observarse a largo plazo.

La aceptabilidad reflejada en el consumo de alimentos se muestra en la Figura 3. Allí se observa que los alimentos con mayores consumos fueron la harina de maíz y la harina de alfalfa, con valores promedios de 57,70 y 53,48 g respectivamente y el menor consumo fue para la harina de coco. Los resultados están en correspondencia con los intentos de consumo analizados anteriormente.

El bajo consumo de este producto por los animales sugiere que, para que puedan ser aprovechados sus excelentes aportes nutritivos, este debe ser mezclado con alimentos más palatables a fin de incrementar su aceptación por los animales. En este sentido, los estudios de Panigrahi (1989) en que se utilizó la harina de coco en raciones de ponedoras comerciales en niveles de 10, 20, 30 y $40 \%$ para evaluar el consumo de alimento, demostraron que las gallinas no presentaron dificultades en consumir y utilizar las dietas que contenían altas concentraciones de harina de coco y mantuvieron normales la producción de huevos y la eficiencia alimenticia.

Vasconcelos y Brandão (1995), estudiando estos mismos niveles de harina de coco en la dieta en pollo de ceba, concluyeron que la utilización de 20 \% de esta harina no afectó la ganancia de peso, el consumo de ración y la conversión de alimento de las aves.

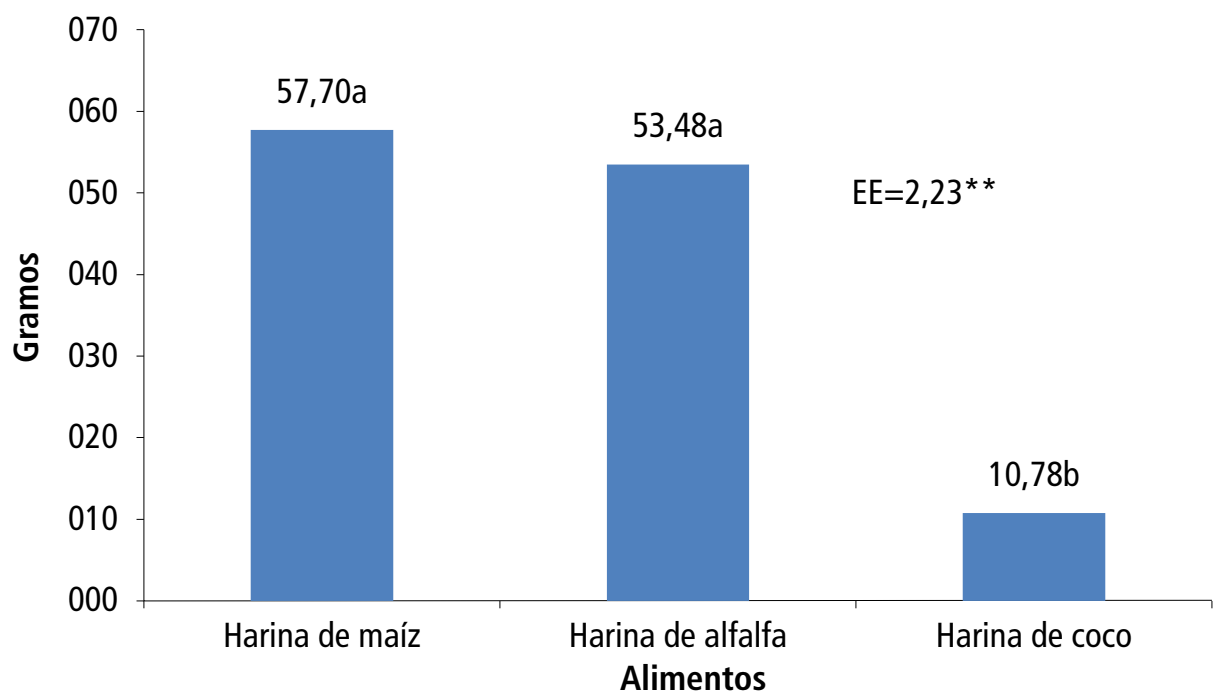

FIGURA 3. Consumo de los alimentos por los conejos en 24 horas de evaluación.

En las dietas en que se incluyó harina de coco desgrasada, los animales realizaron intentos de consumo casi durante las $24 \mathrm{~h}$ (Figura 4), con pico de consumo en los horarios de 15:00 h, 19:00 h y 23:00 h, con descenso importante en los horarios de 1:00 h y 3:00 h, y a partir de las 5:00 h se inicia una 
nueva intensidad de intentos de consumo, es decir, los animales mostraron una tendencia de consumo hacia el horario nocturno.

Esta conducta de consumo de alimento está en correspondencia con resultados similares reportados por Nieves (2004a), quien, al realizar pruebas de aceptabilidad de dietas con niveles crecientes de morera (Morus alba) en conejos destetados, asevera que los conejos incrementan el consumo de alimento en el horario nocturno, coincidiendo además con las horas más frescas, momento en el cual aprovechan más los concentrados y forrajes.

La Tabla 3 muestra el número de intentos de consumo y promedio de consumo de las dietas con niveles crecientes de harina de coco desgrasada. Se observa que los intentos de consumo que mostraron los animales en la dieta que contenía 10 \% fueron los mayores y tuvieron diferencia significativa con respecto a los demás niveles. Por otro lado, en la medida en que aumentó el porcentaje de inclusión de la harina de coco en las dietas por encima del 10 $\%$, los intentos de consumo disminuyeron.

En el caso del consumo de alimento no hubo diferencia significativa entre las dietas del 0 y $10 \%$; sin embargo, existe diferencia significativa con respecto a las demás dietas. Estos resultados demostraron que debido a que los conejos podían comer cualquiera de las cinco dietas, las dietas con 0 y $10 \%$ fueron las más consumidas, lo que comprueba que ellas eran más palatables que las que contenían 20,30 y $40 \%$ de harina de coco.

El consumo de alimento disminuyó a medida que se aumentó el nivel de inclusión de harina de coco en las dietas desde 20 hasta $40 \%$; resultados similares obtuvo Haponik (2009) al utilizar harina de coco en niveles de $25 \%$ de inclusión en la dieta.

Por otro lado, se considera que la menor aceptabilidad de las dietas con 30 y $40 \%$ de harina de coco desgrasada está muy relacionada con el aspecto quemado que se observó en esta, ocasionado por el excesivo calor al que se somete la harina de coco durante su secado industrial, el que puede modificar el aroma, olor y sabor de este producto, haciéndolo menos apetecible para el conejo, animal que presenta papilas gustativas foliadas o foliáceas abundantes y bien desarrolladas; además, en esta especie estas papilas presentan la máxima acumulación de corpúsculos gustativos (Megías et al., 2019).

TABLA 3. Número de intento de consumo y promedio de consumo de dietas con niveles crecientes de harina de coco

\begin{tabular}{|l|c|c|c|c|c|c|}
\hline \multirow{2}{*}{ Indicadores } & \multicolumn{5}{|c|}{ Dietas con \% de inclusión de harina de coco } & \multirow{2}{*}{ EE, sig } \\
\cline { 2 - 7 } & $\mathbf{0}$ & $\mathbf{1 0}$ & $\mathbf{2 0}$ & $\mathbf{3 0}$ & $\mathbf{4 0}$ & s0 \\
\hline Intento de consumo & $6,60^{\mathrm{b}}$ & $9,53^{\mathrm{a}}$ & $3,67^{\mathrm{c}}$ & $2,53^{\mathrm{c}}$ & $2,27^{\mathrm{c}}$ & $0,28^{* *}$ \\
\hline Consumo de alimentos, g & $61,26^{\mathrm{a}}$ & $63,74^{\mathrm{a}}$ & $33,93^{\mathrm{b}}$ & $9,39^{\mathrm{c}}$ & $7,00^{c}$ & $6,26^{* *}$ \\
\hline
\end{tabular}

abc Superíndice con distintas letras en la misma fila indica diferencias significativas a $p \leq 0,01{ }^{* *} 0,01$ 


\section{Conclusiones}

Los conejos tienen mayor aceptación por el maíz y la alfalfa con respecto a la harina de coco desgrasada.

Los animales muestran la mayor aceptación por las dietas del $10 \%$ de inclusión de harina de coco desgrasada, cuando se ofertan libremente en pruebas de aceptabilidad.

Los patrones de consumo descritos demuestran que los conejos consumen alimentos las 24 horas del día, con tendencia a aumentar en el horario nocturno.

\section{Referencias}

De Blas, C., \& Mateos, G.G. (2010). Feed Formulation. In C. De Blas \& J. Wiseman (eds.) Nutrition of the Rabbit (pp. 222-232). CAB International.

Duban J., Gutiérrez N. \& Oviedo O.M. (2012, jul.-dic.). O uso de subprodutos agrícolas na alimentação de coelhos em fase de engorde e reprodução. Biotecnología en el Sector Agropecuario y Agroindustrial, 10(2), 236-242. Duncan, D. B. (1955). Multiple Range Test. Biometrics, 1 1, 1-42. http://doi.org/10.2307/3001478.

Ferreira, W. M., Saad, F. M. O. B. \& Pereira, R. A. N. (2008). Fundamentos da nutrição de coelhos. http://www.coelhoecia.com.br/Zootecnia/ Trabalhos.htm

Haponik, V. C. A., Gastão, B. E., Freitas, E. R., Débora, L. R., Ramos, L. de O. \& De Souza, C. C. (2009). Avaliação nutricional de dietas contendo farelo de coco fornecido a coelhos destinados ao abate. Acta Scientiarum. Animal Sciences Maringá, 31 (4), 357-364.

La O, A.L. (2007). Alimentación de conejos (Oryctolaguscuniculus) con follajes, caña, girasol. (Tesis en para optar al grado científico de doctor en Ciencias Veterinarias). ICA. La Habana. Cuba.

Launchbaugh, L. \& Provenza, F. (1991). Learning and Memory Grazing Livestock Application to Diet Selection. Rangelands, 13(5), 242-244.

Megías M., Molist, P., Pombal, M. A. (2019). Atlas de histología vegetal y animal. Órganos animales. Recuperado (mayo 2020) de: http://mmegias.webs.uvigo. es/2-organos-a/guiada_o_a_inicio.php

Nieves, D. (2005). Forrajes promisorios para la alimentación de conejos en Venezuela. Valor nutricional. En VIII Encuentro de Nutrición y Producción de Animales Monogástricos. Universidad Nacional Experimental Ezequiel Zamora UNELLEZ. Guanare. Venezuela, 17 y 18 de noviembre. http://www.sian.info.ve/porcinos/ publicaciones/encuentros/viiiencuentro duilio.htm.

Nieves, D., Cordero, J., Terán O. \& González, C. (2004a). Aceptabilidad de dietas con niveles crecientes de morera (Morus alba) en conejos destetados. Zootecnia Tropical, 22, 183-190.

Nieves, D., Silva, B., Terán, O., González, C. \& Ly, J. (2004b). A Note on the Chemical Composition and Feeding Characteristics of Diets Containing Leucaena 
leucocephala and Arachis pintoi for Growing Rabbits. Livestock Research for Rural Development 16 (12).

Nieves, D. \& Terán, O. (2006). Uso de recursos arbóreos y arbustivos tropicales para alimentar conejos en Venezuela. Revista Computadorizada de Producción Porcina, 13, suplemento 1.

Nieves, D., Terán, O., Cruz L., Mena, M., Gutiérrez, F. \& Ly, J. (2011). Digestibilidad de nutrientes en follaje de árnica Tithonia diversifolia en conejos de engorde. Tropical and Subtropical Agroecosystems, 14(1), 309-314.

Panigrahi, S. (1989). Effects on Egg Production of Including High Residual Lipid Copra Meal in Laying Hen Diets. British Poultry Science, 30, 305-312.

Stein, H. H., Casas Gloria, A., Abelilla, J.J., Liu, Y. \& Casilda, R.S. (2015). Nutritional Value of High Fiber Co-Products from the Copra, Palm Kernel, and Rice Industries in Diets Fed to Pigs. Journal of Animal Science and Biotechnology, 6(56).

Tamilnadu, J., Moorthy, M., \& Viswanathan, K. (2010). Digestibility and Feeding Value of Coconut Meal for White Leghorn Layers. Veterinary \& Animal Sciences, 6(5), 196-203.

Valverde, D.M. (2010). Usos de la morera (Morus alba) en la alimentación del conejo: el rol de la fibra y la proteína en el tracto digestivo. Agronomía Mesoamericana, 21(2), 357-366.

Vasconcelos, R. Q. \& Brandão, J. S. (1995). Efeito de níveis de farelo de coco na dieta inicial sobre o desempenho de frangos de corte. Revista da Sociedade Brasileira de Zootecnia, Viçosa, 24, 391-400. 